\title{
Abstracts from the ASENT 2010 Annual Meeting March 4-6, 2010
}

\author{
Abstract \#1: EVP-6124 Elicits Pro-Cognitive Effects \\ in Alzheimer's Patients \\ Jeffrey T. Apter \\ Global Medical Institutes, Princeton, NJ
}

Approved symptomatic therapies for Alzheimer's disease (AD) provide modest relief. In the future, it is likely that symptomatic treatments will be utilized with $\mathrm{AD}$ modifying therapies, the development of which are currently a primary focus of research. The nicotinic $\alpha 7$ receptor agonist may be an attractive drug candidate to potentially improve cognition in Alzheimer's disease patients either as a stand-alone therapy or in combination with other symptomatic treatments.

EVP-6124 is a novel, potent, and selective $\alpha 7$ nicotinic receptor agonist. EVP-6124 has an excellent brain to plasma exposure ratio and has shown excellent efficacy and potency in a number of animal models of cognition. Four clinical studies in $>125$ healthy normal human subjects have been completed with EVP-6124, including a single-ascending-dose study, a 14-day multiple-ascending-dose study, a 21-day multiple-dose study, and a single-dose relative bioavailability, food, and gender effect study. EVP-6124 exhibited linear kinetics over the range of 1 to $180 \mathrm{mg}$ and demonstrated a half-life suitable for once daily dosing. EVP-6124 appears to be safe and well tolerated for up to 21 days as measured by adverse events, vital signs, continuous cardiac monitoring, physical examination, and clinical laboratory evaluations. In addition, in normal volunteers, EVP-6124 demonstrated pro-cognitive effects (CogState testing) in various cognitive domains including executive function.

These data suggest that EVP-6124 administered to AD subjects on stable cholinesterase inhibitor therapies may have potential benefit and that further study in this patient population is indicated. Larger phase II studies are currently being initiated in both Alzheimer's disease and schizophrenia.

\section{Abstract \#2: Diffusion Tensor Imaging As a Neuroprotection Outcome Metric in Multiple Sclerosis: Clinical Trial Sample Size Estimates}

Robert J. Fox*, Thomas Cronin ${ }^{\dagger}$, Jian $\operatorname{Lin}^{\dagger}$, Ken Sakaie ${ }^{\dagger}$, Daniel Ontaneda, Shamseldeen Y. Mahmoud, Mark J. Lowe, Michael D. Phillips, and Xiaofeng Wang

*Mellen Center ${ }^{\dagger}$ Cleveland Clinic, Cleveland, $\mathrm{OH}$

Background: Conventional MRI methods detect areas of brain tissue damage, but are poor measures of the degree of injury. Advanced MRI modalities offer imaging metrics of tissue integrity and may be useful in the proof-of-concept evaluation of putative neuroprotective therapies. Previous studies have shown that DTI can be successfully implemented in a multi-center study with good comparability between centers. However, advanced imaging metrics will only be useful if they provide acceptable sample size estimates, which is currently unknown.

Methods: A longitudinal MS patient population was used to estimate the sample size needed to measure a putative neuroprotective therapy. Patients were imaged at baseline, 1, 2, 6, and 12 months. Twenty regions of interest (ROIs) were placed on normal-appearance brain regions from baseline images in each subject and were coregistered longitudinally using FSL and AFNI. A hypothetical neuroprotective therapy was assumed to slow the worsening of DTI measures by $30 \%$ or $50 \%$ relative to those observed in this sample. F tests with multivariate analysis of variance were used to estimate sample size, with correlation over the repeated measured assumed to be 0.85 and $\alpha=0.05$.

Results: For 30\% reduction in FA, 74 subjects per group are needed for $80 \%$ power, and 94 subjects per group for $90 \%$ power. For $50 \%$ reduction in FA, 29 subjects per group were needed for $80 \%$ power, and 36 subjects per group for $90 \%$ power. Additional analyses will include power analyses for other DTI measures and different scanning intervals, as well as additional projections using longitudinal designs models.

Conclusions: Using a longitudinal dataset, feasible sample size estimates are projected for using DTI in a clinical trial of a putative neuroprotective agent. DTI appears to be a feasible candidate marker of neuroprotection in MS clinical trials.

Support: This study was supported by grants from the National Institutes of Health (K23 NS 47211) and the National Multiple Sclerosis Society (RG 3548A2).

\section{Abstract \#3: Converting from CIS to Definite Multiple Sclerosis: An Imaging Based Predictive Model}

Daniel Goldberg-Zimring* ${ }^{\dagger}$, Christian D. Chavarro-Nieto* ${ }^{\dagger}$, Brian C. Healy ${ }^{\dagger}$, Anat Achiron ${ }^{\star}$, Simon K. Warfield, Mohit Neema, Ashish Arora, and Rohit Bakshi

*Laboratory for Neuroimaging Research, ${ }^{\dagger}$ Partners MS Clinical Research Center, Brigham and Women's Hospital, Harvard Medical School; ${ }^{\ddagger}$ Multiple Sclerosis Center, Sheba Medical Center, Tel Hashomer, Israel

Objective: To quantitatively assess brain parenchymal fraction (BPF), gray matter (GM) atrophy, and white matter (WM) lesion load and to determine their association with the likelihood of CIS patients to convert to definite multiple sclerosis (MS) over time.

Background: Patients with clinically isolated syndromes (CIS) are those presenting a first neurologic episode suggestive of MS. A better understanding of the different biomarkers of brain damage in the early stages of MS and their association to clinical progression are essential so that treatments designed to limit or delay tissue damage may be initiated.

Methods: This is a retrospective study of baseline MRI examinations obtained from 12 CIS patients. Eight out of the 12 patients converted to definite MS within 5 years. Demographic characteristics of the patients who converted and did not convert (mean $\pm \mathrm{SD})$ were: age converters $=31.3 \pm 8.6$ and age non-converters $=27.7 \pm 5.2$. Brain MR images were processed by an experienced observer using both semi-automated and manual techniques in order to estimate BPF, thalamus, putamen, caudate, and globus pallidus volumes, as well as PD/T2-weighted, T1-weighted, and Gd-T1w lesion volumes. Comparisons between converters and non-converters were 
based on a Wilcoxon rank sum test. The predictive value of each variable was investigated using a logistic regression.

Results: In those patients who converted, BPF was significantly lower than in those who did not convert $(0.761 \pm$ 0.034 vs. $0.812 \pm 0.016 ; p=0.008$ ). BPF was a perfect discriminator between converters and non-converters. All patients with a BPF $>0.795$ did not convert and all patients with a BPF $<0.795$ converted. We continue to accumulate additional patients for analysis to extend and confirm these findings.

Conclusions: In this preliminary study we found an association between BPF and the likelihood of CIS patients to convert to definite MS. These finding implicate brain atrophy present in the early stages of the disease as playing a role in further disease progression.

Support: This study was supported by research grants from the National Institutes of Health (1R01NS055083-01) and the National Multiple Sclerosis Society (RG4032A1/1, RG3705A1; RG3798A2).

\section{Abstract \#4: Global vs. Regional Spinal Cord Atrophy in Multiple Sclerosis}

Ashish Arora*, Mohit Neema*, Shahamat Tauhid*, Daniel Goldberg-Zimring*, Brian C. Healy,

James M. Stankiewicz, Christian Chavarro-Nieto, Antonia Ceccarelli, Elisa Dell'Oglio, Charles R.G. Guttmann, and Rohit Bakshi

*Laboratory for Neuroimaging Research, Partners MS Center Research Center, Brigham and Women's Hospital, Harvard Medical School, Boston, MA

Objective: To assess whole and regional spinal cord atrophy in patients with multiple sclerosis (MS) using 3T MRI.

Background: Spinal cord damage is common in MS and provides a unique and clinically relevant means to monitor disease progression and therapeutic effects. Whereas regional spinal cord atrophy has been studied extensively in MS, whole spinal cord atrophy has not been previously assessed.

Design/Methods: Whole spinal cord $3 \mathrm{~mm}$ thick T2weighted axial fast spin-echo MRI images were obtained at 3T on 34 patients with MS [26 women and 8 men; 26 relapsing-remitting, 2 clinically isolated syndrome, 4 secondary progressive, 2 primary progressive; Expanded Disability Status Scale (EDSS) $($ mean $\pm \mathrm{SD}) 1.95 \pm 1.72$, range $0-6.5]$ and 15 age-matched normal controls [11 women and 4 men]. The global and regional (cervical, C2-3, and thoracic) cord volumes were determined using a semi-automated tool and were normalized by the segment length and intracranial volume. Spinal cord lesions were also determined using a semi-automated edge finding tool.

Results: Whole cord, C2 -3 , cervical, and thoracic volumes were lower in progressive vs. relapsing patients or normal controls (all $p<0.05$ ). Spinal cord volumes were not related to spinal cord lesion load but were related to EDSS scores $(r=$ -0.35 to $-0.46 ; p<0.05)$. Only the $\mathrm{C} 2-3$ volume correlated with timed 25-foot walk $(r=-0.40 ; p<0.05)$. Whole cord, cervical, $\mathrm{C} 2-3$, and thoracic volumes were all highly intercorrelated $(r=0.83-0.98 ; p<0.05)$.

Conclusions: The established method of estimating spinal cord atrophy, C2-3 cross-sectional volume, provides a useful surrogate of overall global or regional spinal cord atrophy. Spinal cord atrophy is most common in progressive forms of MS and is related to neurologic disability.

Support: This study was supported by grants from the National Institutes of Health (1R01NS055083-01) and the National Multiple Sclerosis Society (RG3705A1; RG3798A2).

\section{Abstract \#5: A Phase I, Dosage-Escalation Study of Creatine Monohydrate in Subjects with Amyotrophic Lateral Sclerosis}

Nazem Atassi*, Eva Maria Ratai*, David Greenblatt ${ }^{\dagger}$, Merit Cudkowicz*, and Allitia DiBernardo

*Harvard Medical School, Boston, MA; ${ }^{\dagger}$ Tufts University School of Medicine, Boston, MA

Background: Mitochondrial dysfunction is a proposed pathologic mechanism in amyotrophic lateral sclerosis (ALS). Creatine has been shown to stabilize the mitochondrial transition pore, buffer intracellular energy stores, stimulate synaptic glutamate uptake, and scavenge reactive oxygen species. It is suggested that creatine is neuroprotective in vivo and that this effect is dose-dependant. Creatine is safe and well-tolerated but failed to demonstrate efficacy in ALS at 5 and $10 \mathrm{~g} / \mathrm{day}$. Experience with creatine in HD patients suggests that creatine may be neuroprotective at higher dosages. The purpose of this study is to establish steady-state serum pharmacokinetics (PK) of creatine at several dosages, and to evaluate the effects of creatine on brain metabolites using proton magnetic resonance spectroscopy $\left({ }^{1} \mathrm{H}-\mathrm{MRS}\right)$

Methods: Six participants with ALS received creatine at three weekly escalating oral dosages (5, 10, and $15 \mathrm{~g}$ BID). Creatine blood levels were collected over 5 hours at each visit. MRS was performed at baseline and 5 days after each dose increase. Pharmacokinetic parameters were estimated by measuring the area under the curve (AUC) for each dosage group. Changes in brain metabolite levels on MRS were compared within subjects before and after treatments using repeated measures RM-ANOVA.

Results To Date: Mean pre-dose steady-state creatine plasma concentrations were 20.3, 39.3, and $61.5 \mu \mathrm{g} / \mathrm{mL}$ at 5, $10,15 \mathrm{~g} \mathrm{BID}$, respectively. Creatine spectra increased by $8 \%$ $(p=0.06)$ and glutamate + glutamine spectra decreased by $17 \%(p=0.039)$ at higher dosages. There were no safety concerns at any of the dosages.

Discussion/Conclusion: Creatine plasma concentrations increased in a dose-dependant manner. Creatine appears to cross the blood-brain barrier, and oral administration of $15 \mathrm{~g}$ BID is associated with increased in vivo brain creatine concentrations and decreased glutamate concentrations. The decrease in brain glutamate concentration with increased creatine dosages is of particular interest given the role of glutamate-mediated injury to motor neurons in ALS. The results of this study support that $15 \mathrm{~g}$ BID creatine could be tested as a therapeutic dosage in ALS clinical trials. These data also support MRS as a potential biomarker for creatine effect on brain metabolites.

\section{Abstract \#6: Magnetic Resonance Disease Severity Scale (MRDSS) for Patients with Multiple Sclerosis: A Longitudinal Study}

Shahamat S. Tauhid*, Jenniffer Moodie ${ }^{\dagger}$, Mohit Neema*, Brian C. Healy $y^{\ddagger}$ Guy J. Buckle, Susan A. Gauthier, Bonnie I. Glanz, Tanuja Chitnis, Samia J. Khoury, Charles R.G. Guttmann, Howard L. Weiner, and Rohit Bakshi

*Laboratory for Neuroimaging Research, Partners MS Center Research Center, ${ }^{\ddagger}$ Brigham and Women’s Hospital, Harvard Medical School, Boston, MA; ${ }^{\dagger}$ University of Massachusetts Medical School, Worcester, MA

Background: We previously described a composite cerebral MRI scale combining T1 lesions, T2 lesions, and whole 
brain atrophy in multiple sclerosis (MS) patients: the Magnetic Resonance Disease Severity Scale (MRDSS).

Objective: Test the strength of the MRDSS vs. individual MRI measures for sensitivity to longitudinal change.

Methods: We prospectively studied 84 patients [age $($ mean $\pm \mathrm{SD}) 42.6 \pm 8.6$ years, EDSS score $3.2 \pm 2.1,68 \%$ $(n=57)$ relapsing-remitting (RR), 24\% $(n=20)$ secondary progressive $(\mathrm{SP})$, and $8.3 \%(n=7)$ primary progressive] over a follow-up period of $3.2 \pm 0.3$ years. Baseline and follow-up brain MRI derived T2 lesion volume (T2LV), T1 hypointense lesion volume (T1LV), and brain parenchymal fraction (BPF). The ratio of T1LV to T2LV assessed lesion severity. MRDSS for each patient was the combination of T2LV, BPF, and T1/T2 ratio. Wilcoxon signed rank test assessed change in each measure over follow-up.

Results: Patients had higher (worse) MRDSS at follow-up $(5.5 \pm 2.3)$ vs. baseline $(4.9 \pm 2.4 ; p<0.001)$. Regarding the individual standardized and unstandardized components of MRDSS, BPF decreased $(p<0.001)$, T1/T2 increased $(p<$ $0.001)$, but T2LV was unchanged $(p>0.5)$. Change in MRDSS was larger than change in individual MRI components. Although MRDSS showed a significant change in both RR (entry $4.4 \pm 2.4$ vs. exit $5.2 \pm 2.3 ; p<0.001$ ) and SP (entry $6.4 \pm 1.8$ vs. exit $6.7 \pm 1.9 ; p<0.05$ ) groups, standardized and unstandardized $\mathrm{BPF}$ and $\mathrm{T} 1 / \mathrm{T} 2$ ratio changed only in the RR $(p<0.001)$ but not in the SP group $(p>0.05)$. Longitudinal change in MRDSS was significantly different between RR and SP groups ( $p=0.002)$; however, the change in the individual standardized and unstandardized MRI components did not differ between groups $(p>0.1)$.

Conclusions: These results suggest the improved sensitivity to longitudinal change of MRDSS vs. individual MRI measures of lesions and atrophy in both RR and SP MS. Although MRDSS shows sensitivity in the progressive phase, it has particular sensitivity in the RR stage of disease. MRDSS shows potential as a tool to monitor disease evolution in MS.

Support: This study was supported by grants from the National Institutes of Health (R01NS055083-01) and the National Multiple Sclerosis Society (RG3705A1 and RG3798A2).

\section{Abstract \#7: Voxel Based Morphometry with DARTEL in Multiple Sclerosis}

Antonia Ceccarelli*, Jonathan S. Jackson*, Ashish Arora*, Shahamat S. Tauhid*, Christian Chavarro-Nieto, Elisa Dell'Oglio, Daniel Goldberg-Zimring, Anshika Bakshi, Rohit Bakshi, and Mohit Neema

*Laboratory for Neuroimaging Research, Brigham and Women's Hospital, Harvard Medical School, Boston, MA

Background: Voxel based morphometry (VBM) is an automated tool used to assess the topography and magnitude of cerebral atrophy from MRI scans. However, VBM output is highly dependent on the input provided by registration and segmentation algorithms. Diffeomorphic anatomical registration through exponentiated lie algebra (DARTEL) is a new tool which has recently been developed to improve registration in healthy volunteers (HV) and neurodegenerative diseases. This has not yet been applied to multiple sclerosis (MS).

Objective: To assess regional gray matter (GM) and white matter (WM) atrophy using VBM combined with DARTEL in MS patients.

Methods: Using a 3T MRI scanner, fluid-attenuated inversion-recovery (FLAIR) and 3D spoiled gradient recalled (SPGR) scans were acquired from 26 relapsing remitting (RR)
MS patients [age (mean \pm SD) $39.2 \pm 9.4$ years, disease duration $8.5 \pm 5.9$ years, Expanded Disability Status Scale (EDSS) score $1.2 \pm 0.9$, FLAIR lesion volume (FLV) $15.0 \pm$ $14.7 \mathrm{~mL}]$ and 28 sex- and age-matched HV. MS lesions on SPGR and FLAIR images were traced using a semi-automated thresholding tool. VBM was performed using DARTEL with statistical parametric mapping (SPM8) software from SPGR images. An analysis of covariance was used to assess differences in GM and WM volume between MS patients and HV. Age, gender, and intracranial volume were included as covariates.

Results: Compared with HV, MS patients showed significant GM atrophy in the bilateral thalami, bilateral caudate, and right putamen. Furthermore, WM atrophy was found in the brainstem, corpus callosum, fornix, and bilateral temporal and occipital lobes. FLV significantly correlated with GM atrophy in the thalami and with WM atrophy in the brainstem, temporal lobes, and corpus callosum.

Conclusions: VBM-DARTEL reveals widespread WM and GM cerebral atrophy affecting many areas of the brain, which is associated with overt lesions in MS patients. This pilot study suggests that VBM-DARTEL is a promising tool to derive meaningful disease specific information in MS.

Acknowledgements: This work was supported by research grants from the National Institutes of Health (NIH R01 NS055083-01) and the National Multiple Sclerosis Society (RG3705A1, RG3798A2).

\section{Abstract \#8: Trial Methodology of a Randomized, Double-blind, Parallel-Group Study Evaluating the Clinical Effectiveness of Carisbamate, Topiramate, and Levetiracetam As Adjunctive Treatment of Partial Onset Seizures in Adults}

Joyce Cramer*, Dennis Dlugos ${ }^{\dagger}$, Frank Wiegand $\$$, Gerald Novak $^{\S}$, William Olson ${ }^{\text {II }}$, and Augusto Grinspan ${ }^{\mathrm{T}}$

* Yale University School of Medicine, New Haven, CT;

${ }^{\dagger}$ Children's Hospital of Philadelphia, Philadelphia, PA; Johnson \& Johnson Pharmaceutical Services, LLC; ${ }^{\S}$ Johnson \& Johnson Pharmaceutical Research \& Development, LLC;

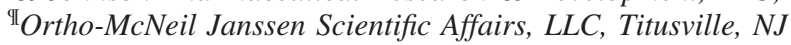

Introduction: International guidelines recommend assessing the overall long-term benefit of antiepileptic drugs (AEDs) using multidimensional measures. Effectiveness encompasses the effects of a product's efficacy, safety, and tolerability. Retention reflects effectiveness over time. This double-blind study (NCT00563459) was designed to compare the 6- and 12-month retention (as the primary parameter) of patients taking carisbamate (the investigational drug), topiramate, and levetiracetam when used as adjunctive treatment for partial onset seizures (POS). The retention hypotheses were that carisbamate would be at least as good as each comparator. Additionally, because cognitive and neuropsychiatric adverse events (AEs) have an impact on acceptance of therapy, the incidences of these AEs were assessed with the hypotheses that carisbamate would be superior to topiramate on cognitive AEs and superior to levetiracetam on neuropsychiatric AEs. The aforementioned hypotheses were analyzed using a fixed-sequence testing procedure.

Methods: Following a screening phase, $\sim 600$ adult patients with POS and a history of inadequate response to treatment with e1AED had to be randomized in a 1:1:1 ratio to enter a 7-week titration period. Target daily doses of study medications were carisbamate $800 \mathrm{mg} /$ day, topiramate $300 \mathrm{mg} /$ day or levetiracetam $2000 \mathrm{mg} / \mathrm{day}$, (dose adjustment could be done 
within the limits of 400-1200 mg/day; $200-400 \mathrm{mg} / \mathrm{day}$; or 1000-3000 mg/day, respectively). After titration, patients entered the 17-week maintenance period. The 7-week titration and 17-week maintenance phases comprise the 6-month (24week) core double-blind phase, with an option for an additional 6-month double-blind extension phase. The primary endpoint was the time from the first intake of study medication to any cause of discontinuation over the 6-month core double-blind phase. Cognitive AEs, neuropsychiatric AEs, reasons for discontinuation, seizure rates, cognitive changes relative to baseline assessed with a computerized cognitive test battery, and patient-reported mood states, behavioral, and cognitive changes relative to baseline using the Profiles of Mood Status questionnaire, Center for Epidemiologic Studies Depression Scale, and patient- and observer-reported Overall Mental Effects Questionnaire among the 3 treatment arms were assessed at 6- and 12 -months post-randomization. This study design can provide unique information and meaningful data that might be valuable for clinical decision-making.

Support: Support was provided by Johnson \& Johnson Pharmaceutical Research \& Development, LLC.

\section{Abstract \#9: Gender Related Outcome Differences after Experimental Mild Traumatic Brain Injury}

\author{
Shihong Li, David J. Loane, Bogdan A. Stoica, and \\ Alan I. Faden \\ Department of Anesthesiology, Shock, Trauma and \\ Anesthesiology Research (STAR) Center, National Study \\ Center for Trauma and EMS, University of Maryland, School \\ of Medicine, Baltimore, $M D$
}

Mild traumatic brain injury (mTBI) has been less well studied than moderate or severe injuries. Emerging clinical evidence from sports related mTBI suggests that females may have worse outcomes than males, yet few animal studies have addressed this issue. The present study compared neurobehavioral and neuropathological outcomes after experimental mTBI in male versus female mice subjected to controlled cortical impact (CCI) brain injury.

Male and female C57BL/6 mice (20-25 g) were subjected to mild CCI (each $n=13$ ) or sham injury (each $n=7$ ). Fine motor co-ordination was evaluated by a beam walk (BW) test at 1 day, 3 days, 7 days, 14 days, and 21 days after injury and cognitive function was assessed using the Morris water maze (MWM) at 15-18 days after injury. T2-weighted MRI was performed on all mice at 21 days after mild CCI, and the injury-mediated lesion volume in each mouse brain was calculated.

Mild CCI caused only a slight increase in the number of foot faults in BW in both male and female mice, without significant gender differences. In the MWM, injured male mice did not show impairments in learning and memory, compared with shams. In contrast, in female mice the latency to locate a submerged platform was significantly increased when compared with either sham or male animals $(p<0.05)$. In addition, MRI showed significantly larger lesion volumes in female mice compared with males at 21 days $(p<0.05)$.

In contrast to long-held views that females may be somewhat protected from brain trauma by virtue of estrogen or progesterone, recent clinical evidence in mTBI suggests otherwise. The current study supports these clinical observations and demonstrates that cognitive impairments are found in female but not male mice following mTBI, with parallel larger lesion volumes. This model may be used to address potential mechanisms that may account for these gender differences.

\section{Abstract \#10: Amyloid Precursor Protein Secretases As Therapeutic Targets for Spinal Cord Injury}

\author{
Ahdeah Pajoohesh-Ganji* and Alan Faden ${ }^{\dagger}$ \\ *Georgetown University, Washington, DC; ${ }^{\dagger}$ University of \\ Maryland School of Medicine, Baltimore, MD
}

Spinal cord injury (SCI) has a prevalence of 250,000 in the United States and an incidence of 12,000 cases per year. Secondary biochemical and physiological changes induced by trauma lead to delayed cell death, inflammation, glial scar formation, and impaired regeneration. Identification of secondary injury processes may lead to development of new therapies to limit tissue damage and improve functional outcome.

Amyloid precursor protein (APP) is upregulated after SCI with a peak at 3 days. APP is sequentially cleaved by $\gamma$-secretase and $\beta$-secretase (BACE1) to produce $\mathrm{A} \beta$, which is directly neurotoxic and also serves to activate microglia by releasing associated neuroinflammatory factors. We hypothesized that $\mathrm{A} \beta$ increases after $\mathrm{SCI}$ and contributes to secondary damage, and that treatment with a $\gamma$-secretase inhibitor will reduce inflammation, limit tissue loss, and reduce the functional deficits after injury.

Here we show that $A \beta$ increases at 1 day, peaks at 3 days, and begins to decrease at 7 days after spinal cord injury in mice. PS1, which is a component of $\gamma$-secretase, and BACE1 are upregulated after SCI. Moreover, treatment with a $\gamma$-secretase inhibitor (DAPT) reduces the production of $\mathrm{A} \beta$ after injury. These data suggest that $\gamma$-secretase inhibitors may have therapeutic benefit after SCI.

Support: This work was supported by grants from the Paralyzed Veterans of America (PVA2685) and the National Institutes of Health (5R01NS054221-03).

\section{Abstract \#11: The Disruptions to Cognition, Everyday Function, and Quality of Life in Oncology Patients: A Therapeutic Opportunity?}

Keith Andrew Wesnes, Helen Brooker, and Chris Edgar
United BioSource Corporation, Goring on Thames, UK

Introduction: Patents with a variety of forms of cancer often report cognitive difficulties, and cognitive testing has confirmed the presence of impairments to attention and memory in several oncology populations. The purpose of this evaluation was to assess the profile of impairments across major cognitive domains, and relate this to a variety of factors including quality of life (QOL), everyday function, cancer type, age, depression, and anxiety.

Methods: The study population comprised 432 females (mean age 57.6 years; range 18 to 86 ) and 456 males (mean age 60.6 years; range 18 to 88 ), diagnosed with either Hodgkin's disease, non-Hodgkin's lymphoma, chronic lymphocytic leukemia, or multiple myeloma. An automated cognitive test battery (the CDR System) was administered which assessed core aspects of cognition including attention, working and episodic memory. The ECOG Performance Status Score, the FACT-AN, and the Cancer Linear Analog Scales (CLAS) were also administered. The CDR System normative data base of over 6000 healthy individuals was used to determine the cognitive deficits using Cohen's $d$ effect sizes in five age cohorts. Functional ability on the ECOG was related to cognitive function in these age cohorts, and the relationships between cognitive function and various QOL measures were also examined.

Results: Patients in all age bands showed marked impairments to the Power of Attention measure from the CDR System, with large effect sizes, together with notable slowings in the speed of retrieval of information from memory. The older 
patients also showed deficits in other aspects of attention and working and episodic memory. There were clear associations between cognitive function and various quality of life indices, and a good overall relationship was established between the ECOG Performance Status Score and cognitive function; the strength of this relationship increased with age.

Conclusions: There is a clear cognitive deficit profile in cancer patients, which increases both in range and magnitude with aging. The strong relationship of the CDR attentional measures to everyday function and QOL is consistent with findings in other clinical populations (e.g., dementia). Cognitive dysfunction is a therapeutic target in many diseases, and the deficits identified here suggest that treatment would be valuable in oncology. Early results with modafinil and epoetin alfa have been encouraging in this respect.

\section{Abstract \#12: Computerized Essential Tremor Spirography: Development of a Rater-Independent Outcome Measure for Clinical Trials}

Dietrich Haubenberger*, Daniel Kalowitz ${ }^{\dagger}$, Fatta B. Nahab ${ }^{\ddagger}$, Camilo Toro*, Daniel Ippolito ${ }^{\text {II }}$, Loretta Wittevrongel*, and Mark Hallett*

*National Institutes of Health, Bethesda, MD; ${ }^{\dagger}$ University of Maryland, College Park, MD; ${ }^{\ddagger}$ University of Miami, Miami, FL; " Duke University, Durham, NC

Action tremor represents the cardinal symptom of essential tremor (ET) impairing patients' activities of daily living such as handwriting. Although visually rated Archimedes spirals are used to document tremor intensities, computerized methods of spiral analysis have been presented to quantify ET tremor. Here, we describe a method to objectively quantify tremor using digitizing tablet-based spirography and compare the measures with standardized visual rating after treatment intervention.

Nine ET patients underwent a standardized ethanol challenge. Spirals were drawn at six time-points (baseline, 15, 30, 45,60 , and 75 min post-ethanol) using a digitizing tablet. Two methods for data-selection were applied (T: fixed number of time-points of the time-series, S: fixed space by capturing 3.75 spiral turns). After numerical differentiation of the time series, velocity spectra were calculated. Velocity at the spectral peak frequency was used as measure of tremor amplitude. As reference method, visual ratings (VR) were obtained from seven raters using a standard rating scale. Per method, values were normalized to baseline. Larger amplitude changes between time-points were considered as higher sensitivity in demonstrating changes after treatment intervention. Repeated measures ANOVA was used to calculate differences between methods, with Bonferroni correction for post hoc comparisons.

For each method, repeated measures ANOVA showed a significant time effect, demonstrating a reduction in tremor amplitudes after ethanol intake (VR: $p=0.001$; $\mathrm{T}: p=0.008$; $\mathrm{S}: p=0.003)$. After normalizing to baseline, the method effect across time-points was significant ( $p=0.001)$, as well as factor time $(p=0.002)$. Method $\mathrm{S}$ exhibited larger changes from baseline across time-points $(p=0.041)$, whereas method T showed no significant differences compared with VR.

Ethanol significantly reduced tremor amplitudes across all methods. Computerized method $\mathrm{T}$ equally documented change across time-points as VR; method $\mathrm{S}$, however, showed a significantly higher sensitivity to change, compared with the reference method. In conclusion, the proposed methods of spiral analysis are either equally sensitive (method T) or more sensitive (method S) to treatment-induced changes of tremor intensity and therefore might be useful as outcome measures in clinical trials in ET.

\section{Abstract \#13: Low-Dose Naltrexone's Tolerability and Effects in Fatigued Patients with Parkinson's Disease: An Open-Label Study}

Thomas Guttuso, Jr., Naomi Salins, and David Lichter State University of New York at Buffalo

Introduction: One third of idiopathic Parkinson's disease (IPD) patients report fatigue as their most disabling symptom, and $58 \%$ consider fatigue to be one of their three most disabling symptoms. The use of low-dose naltrexone (LDN), $4.5 \mathrm{mg}$ qhs, has been anecdotally reported to improve fatigue in IPD.

Methods: We are examining the tolerability and effectiveness of LDN in patients with parkinsonism in an ongoing openlabel trial. Fatigue and motor symptoms in the "On" state are being assessed at baseline and every 4 months over 1 year using the Parkinson Fatigue Scale (PFS-16) and the Unified Parkinson's Disease Rating Scale (UPDRS), respectively. Global Satisfaction of Treatment is also being assessed. We report here on the changes in fatigue and motor scores among the 4 IPD subjects who were fatigued at baseline, defined as a score of e 53 on the PFS-16. We also compare the fatigue changes with those from previous trials in fatigued IPD and multiple sclerosis (MS) patients.

Results: Eight subjects have been enrolled since January 2009. The 4 IPD subjects who were fatigued at baseline have experienced a mean $22 \%$ reduction in PFS-16 scores (range $13-30 \%$ ) and a $15 \%$ increase in total UPDRS "On" scores after 8 months of LDN therapy. Mean Global Satisfaction of Treatment at 8 months was 8.75 on the 10 -point scale. No side effects have been associated with LDN therapy among the 8 subjects. Based on previous randomized controlled trials (RCTs), the only effective therapy for fatigue in IPD has been methylphenidate, which showed a $16 \%$ decrease in fatigue from baseline. The three therapies demonstrating effectiveness in treating fatigue in MS patients in RCTs have been amantadine, modafinil, and aspirin, which decreased fatigue by $24 \%$, $20 \%$, and $18 \%$ from baseline, respectively. Modafinil was shown to be ineffective in treating fatigue in IPD.

Conclusions: In this small, open-label trial, LDN therapy was well tolerated and was associated with equivalent reductions in fatigue compared with historical benchmarks. These observed reductions in fatigue are unlikely to have been a function of any perceived improvements to motor symptoms, because UPDRS scores slightly worsened over the 8-month trial, as expected in this progressive disease.

\section{Abstract \#14: Development of a Novel Non-Anxiogenic Broad Spectrum Anti-Emetic}

Elaine Alexander*, E.H. Pfadenhauer*, Charles von Gunten ${ }^{\dagger}$, and David Helton

*Cenomed, Inc.; ${ }^{\dagger}$ Institute for Palliative Medicine, San Diego, $C A$

Introduction: There remains an unmet medical need for more efficacious anti-emetic agents with fewer side effects for the treatment of nausea/emesis occurring in patients with terminal, advanced, or chronic diseases, cancer patients receiving chemo- and/or radiotherapy, and motion sickness in civilian and military settings. The discovery and development of new compounds with a novel receptor profile for the improved control of nausea/emesis is of considerable scientific interest and clinical relevance.

Methods: CR-2526, a candidate small molecule identified in Cenomed Research's multi-neurotransmitter receptor screening program, was administered in vivo to two species (cat/ 
shrew) in three validated emesis models: chemical (xylazine), cisplatin, and motion.

Results: CR-2526 decreased the number of emetic events, prolonged latency, and eliminated animal behavior interpreted to be associated with the distress of nausea in each of the three emesis models in both species in a dose-dependent manner. Representative data for cisplatin-induced emesis in shrews are presented here. The mean number of emetic events per animal $(n=5)$ was $0.2 \pm 0.06$ in the CR-2526 treated group versus 2.8 \pm 0.7 in the control group $(p<0.05)$. The mean latency to emesis was $87.8 \pm 1.9 \mathrm{~min}$ in the CR-2526 group compared with $27.7 \pm 5.3 \mathrm{~min}$ in the control group $(p<0.05)$. At the end of the 90-min observation period, four of the five animals in the CR-2526 group had not yet had an emetic event.

Pharmacological evaluation suggests that the anti-emetic efficacy is due to 5-HT1A receptor agonism and additional activity at least one other neurotransmitter receptor, not previously implicated in nausea/emesis. CR-2526 appears to have a benign side effect profile, including the absence of induction of either anxiety (defensive behavior) or sedation, with a wide therapeutic ratio of 60:1 (side effects/efficacy).

Conclusions: CR-2526 is a promising potential clinical candidate for the effective treatment of nausea/emesis induced by a broad spectrum of emetic challenges in a wide range of clinical indications without undesired side effects of sedation, anxiety, or induction of panic attacks. Based on pre-clinical data, anticipatory nausea also may be a clinical target. CR-2526 appears to act through 5-HT1A agonism and through a newly identified receptor in the final common central pathway for nausea/emesis. The compound could provide effective treatment for inadequately controlled nausea/emesis and potentially replace the multi-pharmacology often required in multiple clinical settings. The compound is on a pre-IND path with plans to execute a phase I/II study in humans.

Support: Cenomed, Inc. sponsored these investigations.

\section{Abstract \#15: Efficacy and Safety of Long-Acting Injectable Paliperidone Palmitate Relative to Long- Acting Injectable Haloperidol, Bromperidol, and Fluphenazine Decanoate for Long-Term Treatment in Patients with Schizophrenia Using Number Needed to Treat and Number Needed to Harm}

Srihari Gopal*, Joris Berwaerts*, Isaac Nuamah*, Kasem Akhras ${ }^{\dagger}$, Danielle Coppola, Ella Daly, David Hough, and Joseph Palumbo

*Johnson \& Johnson Pharmaceutical Research \& Development, LLC; ${ }^{\dagger}$ Johnson \& Johnson Pharmaceutical Services

Background: Atypical antipsychotic long-acting injectables (LAIs) such as paliperidone palmitate (PP) are believed to provide a favorable safety profile compared with typical LAIs. Because no trials have compared PP directly with typical LAIs, we assessed the benefit-risk of PP relative to typical LAIs using published placebo-controlled trials.

Methods: A computerized search in MEDLINE was conducted. Number needed to treat (NNT), number needed to harm $(\mathrm{NNH})$, and the likelihood of being helped or harmed (LHH) were calculated from published placebo-controlled studies of PP and the following decanoate LAIs: haloperidol (HD), bromperidol (BD), and fluphenazine (FD). NNTs were calculated for prevention of psychotic relapse. NNHs were calculated for extrapyramidal symptoms: akathisia, tremor, and tardive dyskinesia reported as an adverse event, anticholinergic use, and positive Abnormal Involuntary Movement Scale (AIMS) total score.

Results: NNTs for relapse were similar for all LAIs: 2-5. NNHs varied considerably: akathisia 205 (PP) vs. 10 (BD); tremor 69 (PP) vs. -5 (BD); anticholinergic use 30 (PP) vs. 5 (FD); AIMS positive score -33 (PP) vs. 13 (FD). For tardive dyskinesia the NNH was infinity for PP vs. 7 for FD. LHH for preventing relapse vs. anticholinergic use was 15 for PP and 2.5 for FD.

Conclusions: Effect size as measured by NNT for relapse was similar across different LAIs. NNHs for extrapyramidal symptoms and related events were lower for typical LAIs, compared with PP. Although the benefits in prevention of relapse are similar among LAIs, the risks of extrapyramidal symptoms vary considerably. PP has a favorable LHH compared with typical LAIs.

\section{Abstract \#16: Optical Coherence Tomography As a Clinical Trial Outcome Measure in Multiple Sclerosis: Implications of Treating On-Study Relapses with Intravenous Methylprednisolone}

\author{
Robert Bermel, Peter Imrey, and Jeffrey Cohen \\ Cleveland Clinic, Cleveland, $\mathrm{OH}$
}

Background: Optical coherence tomography (OCT) is potentially useful as an outcome measure for clinical trials of neuroprotective therapies in multiple sclerosis (MS). In largescale clinical trials, it is common for study participants to receive intravenous methylprednisolone (IVMP) as a treatment for relapses while on study. Other anatomic imaging measures such as brain atrophy are subject to transient changes attributed to the anti-inflammatory and water-shifting effects of IVMP, which necessitates delaying imaging studies within a study relative to IVMP dosing. We studied whether IVMP induces short-term changes in OCT metrics including retinal nerve fiber layer thickness (RNFLT) and macular volume (MV).

Methods: $20 \mathrm{MS}$ patients who received treatment with IVMP for a relapse other than optic neuritis plus 11 controls (patients with MS without relapse or IVMP treatment) were prospectively recruited. Baseline Stratus OCT Fast RNFLT and Fast Macular Volume protocols were performed immediately prior to the first dose of IVMP and repeated 14 days later in steroid-treated patients. Control patients not receiving IVMP underwent follow-up OCT between 14 and 28 days after the baseline scan. A standard protocol of $1000 \mathrm{mg}$ IVMP daily for 3 days followed by a 12-day oral prednisone taper was utilized in all steroid-treated patients. Patients on intermittent pulse IVMP were excluded. Repeated measures mixed statistical models were used to estimate the effect of treatment with IVMP on RNFLT and MV.

Results: Data from one eye of a control participant were excluded because unilateral primary retinal pathology invalidated the OCT data. All other data were analyzed, including baseline data from one patient in each group who did not complete followup. Mean RNFLT at baseline was $87.9 \mu \mathrm{m}$ in steroid-treated participants and $81.9 \mu \mathrm{m}$ in control participants. Mean baseline to follow-up change in RNFLT in steroid-treated patients was 2.80 $\mu \mathrm{m}$ (range -4.76 to $17.79 \mu \mathrm{m}$ ). Based on the mixed effects model, the estimated treatment effect of IVMP on baseline to follow-up RNFLT change was $2.14 \mu \mathrm{m}(95 \% \mathrm{CI}=-1.63$ to 5.92; $p=0.25$ ). For MV change, the estimated treatment effect was $0.11 \mathrm{~mm}^{3}$ (95\% CI $=-0.05$ to $0.28 ; p=0.17$ ).

Conclusions: The observed trends suggest any treatment effect of a single course of IVMP on RNFLT and MV is small, and that altering the timing of OCT following treatment of MS relapses within clinical trials may not be necessary. Additional data are needed to confirm this. 